Corresponding Author: Suhair Abdelrahim Osman Hassan;

email:

othman_suhair@outlook.com

Received 12 November 2020

Accepted 25 December 2020

Published 31 December 2020

Production and Hosting by

Knowledge E

(c) Suhair Abdelrahim Osman Hassan. This article is

distributed under the terms of the Creative Commons

Attribution License, which permits unrestricted use and redistribution provided that the original author and source are credited.

Editor-in-Chief:

Prof. Mohammad A. M. Ibnouf

\section{Treatment of Acute Bronchiolitis in a Resource-limited Setting in Port Sudan City: A simple Effective Regimen}

\section{Suhair Abdelrahim Osman Hassan}

Associate Professor, Consultant Paediatrician and Neonatologist, Port Sudan Paediatric Teaching Hospital, Red Sea University, Sudan

\section{Abstract}

Background: Bronchiolitis is an acute lower respiratory viral infection, caused by the Respiratory Syncytial Virus (RSV) in $60-85 \%$ of cases and other respiratory viruses in remaining ones. It is the leading cause of hospitalization for infants and toddlers during the winter and early spring months. At the moment, there is no consensus on its treatment worldwide. The prevalence is increasing in port Sudan eastern Sudan, while we have no admission policy or a unified management protocol. Therefore, the aim of this study is: (1) to formulate an admission policy and a new simple management protocol; (2) to determine the factor(s) that increased the prevalence in port Sudan as a secondary objective; and (3) to compare the outcome in the study (119 infants) and historical (135 infants) groups concerning the hospitalization length and death rate as a primary objectives.

Method: This is a hospital-based, controlled clinical trial (CCT) study, demographic data were collected by a questionnaire, and data were analyzed manually. Severity criteria for acute bronchiolitis are defined. A management guideline was defined and applied to bronchiolitis cases (119 case) with intention to treat, from October to April each year (2013-2015). All infants aged between 1 and 23 months with only acute bronchiolitis were included, while cases with bronchiolitis and associated dysmorphic features, heart disease, tracheo-oesophageal fistula, other respiratory diseases, other morbidities, infants below one month or above two years were excluded from this study. The outcome was compared to a group of matched infants with bronchiolitis (135 cases) who received antibiotics, B2 agonist, and steroids, between October and April of years 2011-2012.

Results: While several new lifestyle factors increased the prevalence, the new guidelines decreased the admission rate (9.6 vs $5.7 \mathrm{mo}$ ), is simple and effective, less expensive, and the duration of hospitalization also reduced $(p=0.04)$.

Keywords: acute bronchiolitis, resource-limited settings, advanced respiratory intervention, simple effective intervention,S-7Pro3NO regimen 


\section{Introduction}

Bronchiolitis is the leading cause of hospital visits and admission among infants and toddlers during the winter and early spring months [1], causing small airways obstruction and air trapping [2-4], especially among urban population living in flats or apartments, due to limited space, closer contact, and decreased house ventilation rather than those living in large houses with compounds or villas. Some cultural factors also play a role in the prevalence and severity of the disease, such as avoiding bathing infants in winter months in order to prevent them from cold or the lack of knowledge on the importance of hand washing. Many viruses like Metapneumovirus, Adenovirus, Influenza and Para influenza, Rhinovirus and human bocavirus are accused, but the most common one is the Respiratory Syncytial Virus (RSV) [1, 2]. Although the peak age for bronchiolitis is three-six months, it mostly occurs in infants less than four months old, who may need advanced respiratory support with CPAP and mechanical ventilation [2-6], which is not always attainable in developing countries and form an obstacle to manage such cases. Severity is variable - from a mild disease that needs simple intervention and follow-up (happy wheezers) to a severe one that necessitates intensive care. Fortunately, in Sudan, severe cases are less common and most cases are mild to moderate. This is attributed to the wide, well-ventilated houses, and the abundant sun. There is no specific treatment. Antibiotics, bronchodilators, and corticosteroids are controversial [2, 4]. Though there is no consensus between different institutes and even between consultants in the same hospital, concentrated saline ( $\geq 3 \%$ ) nebulization gained the consensus [5-12]. Most units use $\mathrm{O}_{2}$, short $\beta 2$ - agonists (salbutamol), antibiotics, and hydrocortisone, resulting in prolonged hospitalization, morbidity, staff work burden, and parents' dissatisfaction.

Moreover, in a resource-constrained settings where intensive care facilities are limited, ways to save lives of infants with severe bronchiolitis should be obtained and implemented. So, the aim of this study is to (1) formulate an admission policy depending on the disease severity; (2) create a simple management protocol as there is no known management protocol for bronchiolitis in Sudan; (3) determine the factor(s) that increased the prevalence in port Sudan as a secondary objective; and (4) compare the outcome in the study (119 infants) and historical (135 infants) groups concerning the hospitalization length and death rate as a primary objectives.

\section{Methods}

This is an observational, longitudinal study comparing the outcome in the cases of moderate and severe bronchiolitis, in patients aged 1-23 months, admitted to the Sea Ports Corporation Hospital (SPCH) in Port Sudan, Sudan, during the winter months (October-April) of the years 2013-2015 (a total of 119 infant - 9 severe and 110 moderate cases). Hospital records of all admitted bronchiolitis cases in the years 2011-2012 were revised, 137 cases were found, 2 were excluded due to associated bacterial pneumonia in one and dysmorphic features in the other. The remaining 135 infants $(7$ severe, 128 moderate) in the same age range were compared to the study group. Data were collected and spread on an Excel sheet and analyzed manually. 
To unify the severity and admission criteria and classification of cases, the following definitions are made (see Table 1):

1. Severe case is defined by the author as an infant who is sick with spasmodic cough, tachypnoea (RR $60 \mathrm{bpm}$ or more), severe retractions of the sub-costal area and tracheal tug, nasal flaring, lethargic or irritable, unable to feed and has typetwo respiratory failure on blood gases or clinical cyanosis while breathing room air or hypoxic (SPO2 $\leq 90$ in room air), with inspiratory and expiratory rhonchi on lung auscultation.

2. Moderate case is defined by the author as an infant who is unwell, has a spasmodic cough, tachypnoea (RR $50-<60$ bpm), with retractions, decreased feeding and type-1 respiratory failure on blood gases or hypoxia (SPO2 90-94\% while breathing room air), with inspiratory and expiratory rhonchi on lung auscultation.

3. Mild case is defined by the author as an infant who is well with spasmodic cough but no tachypnoea ( $\mathrm{RR} \leq 50 \mathrm{bpm}$ ), has mild or no retractions, pink (SPO2 $\geq 96$ $\%)$ while breathing room air and feeding well with rhonchi on lung auscultation.

TABLE 1: Definition of the severity phenotype.

Criterion
General condition
RR (bpm)
Retractions
SPO2\% in room air
Cyanosis
Blood gases
Ability to feed

\begin{tabular}{|c|}
\hline Severe \\
\hline Lethargic/irritable \\
$\geq 60$ \\
Severe \\
$\leq 90$ \\
++ \\
\hline Type $11 \mathrm{RF}$ \\
Unable
\end{tabular}

\begin{tabular}{|c|}
\hline Moderate \\
\hline Unwell \\
\hline $50-<60$ \\
\hline Present \\
\hline $90-94$ \\
$+/-$ \\
\hline Type 1 RF \\
\hline Decreased \\
\hline
\end{tabular}

\begin{tabular}{|c|}
\hline Mild \\
\hline Well \\
\hline$<50$ \\
\hline Mild/no \\
$\geq 96$ \\
\hline- \\
\hline Normal \\
\hline Normal
\end{tabular}

A new simple, supportive regimen is defined and implemented by the author, and applied to all admitted cases (total $=119$ case) with intension to treat.

Reg-1 (for severe cases) composed of:

1. Provide vital signs assessment every 2 hr or as needed;

2. Provide nebulized $3 \%$ saline or $\mathrm{N} / \mathrm{S}$ through a high flow $\mathrm{O}_{2} \geq 8 \mathrm{~L} / \mathrm{min}$, continuously or every $1 \mathrm{hr}$ till stabilization;

3. Provide L-epinephrine nebulizer $2.5 \mathrm{ml}$ diluted in $5 \mathrm{ml} \mathrm{N} / \mathrm{S}$, every $2-4 \mathrm{hr}$;

4. Provide Oxygen as needed to maintain $\mathrm{SPO} 2 \geq 96 \%$;

5. Provide I.V. fluids, 2/3rd of the maintenance, and keep NPO;

6. Provide NGT mucolytic syrup $1.5-2.5 \mathrm{ml}$ every $8 \mathrm{hr}$, depending on age;

7. Provide nasal care (congestion/obstruction relievers like Sea water nasal spray \pm nasal drops containing ephedrine, plus nasal suctioning when needed); 
8. No antibiotics;

9. No steroids; and

10. No $\beta 2$ agonist.

Reg-2 (for moderate cases) composed of:

1. Provide vital signs assessment every $2 \mathrm{hr}$;

2. Provide $10 \mathrm{ml}$ nebulized $3 \%$ saline or N/S through a nebulizer, every $2-4 \mathrm{hr}$;

3. Provide L-epinephrine nebulizer $2.5 \mathrm{ml}$ diluted in $5 \mathrm{ml} \mathrm{N} / \mathrm{S}$, every 4-6 $\mathrm{hr}$ if needed;

4. Provide Oxygen as needed to maintain $\mathrm{SPO} 2 \geq 96 \%$;

5. Provide NGT feeds or I.V. fluids if needed or allow direct sucking if possible;

6. Provide oral mucolytic syrup $1.5-2.5 \mathrm{ml}$ every $8 \mathrm{hr}$, depending on age;

7. Provide nasal care (congestion/obstruction relievers [sea water sprays] \pm nasal drops containing ephedrine, plus nasal suctioning when needed);

8. No antibiotics;

9. No Steroids; and

10. No $\beta 2$ agonist.

Control group received salbutamol nebulizer, $\mathrm{O}_{2}$, antibiotics, steroids, and fluids or NGT feeds plus saline nasal drops. The first group (study group) received Reg-1 regimen (for severe cases), and Reg-2 regimen (for moderate cases).

\subsection{Study group}

All patients (1-23 months old) to were admitted to the pediatric wards of the Sea Ports Corporation Hospital (SPCH) in Port Sudan, Sudan with bronchiolitis in the winter months (October-April) of the years 2013-2015 and received the new regimen without antibiotics, steroids, or salbutamol nebulizer. The exclusion criteria of the study were any congenital abnormalities including heart, syndromic infants, and other associated medical problems or evidence of respiratory bacterial infection. Mild bronchiolitis cases were followed at the outpatient clinic.

Severe cases were admitted directly to ICU and received continuous, either $3 \%$ saline or N/S (if 3\% is not available) nebulization, that is, we put 20 cc NS or $3 \%$ saline in the nebulizer cub and attach it to a humidifier (bubbler) that attached to an oxygen cylinder, set at $8 \mathrm{~L} \mathrm{O}_{2}$ or more/min, and the amount of saline was replaced immediately whenever finished. Adrenaline (L-epinephrine) $2.5 \mathrm{cc}$ (two and half ampoules of $1 \mathrm{mg} / 1 \mathrm{ml}$ ) diluted in $5 \mathrm{ml} \mathrm{N} / \mathrm{S}$ was given as nebulizer every $2 \mathrm{hr}$, through a face mask, with continuous monitoring of vital signs. All infants were kept nothing per mouth (NPO), given two-thirds of their maintenance fluid, plus nasal care with sea water spray (hypertonic saline) as 
needed, ephedrine containing nasal drops for children every $8 \mathrm{hr}$, plus nasal suctioning as needed (Reg-1. Infants from this group who improved to moderate state were shifted to the pediatric ward to continue their treatment of Reg-2).

Moderate cases were admitted to the pediatric ward and received intermittent N/S or $3 \%$ saline (every 1-2 hr) nebulization, plus diluted adrenaline nebulization same dose as above but at a 3-4 hr interval, plus I.V. fluid or NGT feeds, plus same nasal care and monitoring. Those who improved and reclassified as mild were discharged home with follow-up.

\subsection{Historical control group}

This group comprised of 135 patients ( 7 severe, 128 moderate cases), all of them received the same treatment regimen of: antibiotic ceftriaxone plus $\beta 2$ agonist nebulization every 4-6 hr plus injectable steroid (hydrocortisone) and other supportive care (I.V. fluids or NGT feeds, $\mathrm{O}_{2}$ /nasal cannula, and NS nasal drops \pm nasal suctioning).

\section{Results}

With the application of the new Regimen, the admission rate decreased significantly (39.7/season vs 67.5/season, $P<0.5$ ).

In the study group of 119 infants with bronchiolitis, 9 cases (7.6\%) were severe, 110 (92.4\%) were moderate and no deaths were encountered (Table 2 ) .

TABLE 2: Severity phenotype and outcome in the study group.

\begin{tabular}{|l|c|c|c|}
\hline Type & Discharged & Died & Total \\
\hline Severe & $09(100 \%)$ & 0 & 9 \\
\hline Moderate & $110(100 \%)$ & 0 & 110 \\
\hline Total & 119 & 0 & 119 \\
\hline
\end{tabular}

TABLE 3: Severity phenotype and outcome in the control group.

\begin{tabular}{|l|c|c|c|}
\hline Type & Discharged (\%) & Died (\%) & Total \\
\hline Severe & $03(42.9)$ & $04(57.1)$ & 7 \\
\hline Moderate & $125(97.7)$ & $01(0.8)$ & 128 \\
\hline Total & $128(94.8)$ & $05(3.7)$ & 135 \\
\hline
\end{tabular}

In the historical control group of 135 infants, 7 were severe and 128 moderate. The death rate was $3.7 \%$ (Table 3 ) with statistically significant difference from the study group $(P<.04)$

The mean hospitalization time in the study group was 6.4 days in severe cases and 4.7 days in the moderate group, while it was 11.1 days in severe cases and 8 days in moderate cases in the control group, which is again, statistically significant $(P<0.5)$ (see Table 4).

Table 5 summarizes the major differences between the two groups. 
TABLE 4: The mean hospital stay.

\begin{tabular}{l|c|c|c|}
\hline MHS* (days) & Study $\left(\mathbf{( 1}^{\text {st }}\right)$ group & Control $\left(\mathbf{2}^{\text {nd }}\right)$ group & P-value \\
\hline Severe cases & 6.4 & 11.1 & $<0.5$ \\
\hline Moderate cases & 4.7 & 8 & $<0.5$ \\
\hline
\end{tabular}

*Mean Hospital Stay

TABLE 5: Comparison between the outcomes in the two groups.

Observation
Total no. of cases
Severe cases
Moderate cases
Discharged
Deaths

\begin{tabular}{|c|}
\hline Study group \\
\hline 119 \\
\hline $09(7.6 \%)$ \\
\hline $110(92.4 \%)$ \\
$(100 \%) 119$ \\
\hline $0(0 \%)$ \\
\hline
\end{tabular}

\begin{tabular}{|c|}
\hline Control group \\
\hline 135 \\
\hline $07(5.2 \%)$ \\
\hline $128(94.8 \%)$ \\
\hline $128(94.8 \%)$ \\
\hline $5(3.7 \%)$ \\
\hline
\end{tabular}

Many factors are found to contribute to the increasing prevalence of bronchiolitis in Sudan (Table 6 ). Interestingly, $100 \%$ of the carers believed that bath in the winter will subject/increase the flu illness, while more than $98 \%$ were not aware of the importance of hand washing. About $71 \%$ of the cases were residing in flats or unventilated houses, while $90 \%$ were unaware of the importance of opening widows to allow sun and air exchange. In $88 \%$ of the cases, the families were large, and $73 \%$ of the mothers were either illiterate or had received low-grade education, while in $53 \%$ there was a smoking person in the house (see Table 6)

TABLE 6: Contributory factors to bronchiolitis

\begin{tabular}{l|c} 
Contributory factor & $\%$ \\
\hline Avoiding bathing infants during the winter months & $100 \%$ \\
\hline Unawareness of the importance of hand washing & 98.55 \\
\hline $\begin{array}{l}\text { Contact with a household common cold/flu case } \\
\text { Unaware of the importance of allowing sun rays and air exchange } \\
\text { (opening the widows) in the house }\end{array}$ & $97 \%$ \\
\hline Large families and overcrowding & $90 \%$ \\
\hline Illiterate/low-grade educated mothers/parents & $88 \%$ \\
\hline Residence in flats/apartment or a small house & $73 \%$ \\
\hline A smoking person inside the house & $71 \%$ \\
\hline
\end{tabular}

The application of the stated definitions for the disease severity decreased the admission rate (39.7/season vs 67.5/season, $P<0.5)$, thereby decreasing the burden on the staff and doctors.

The protocol was found to be simple, satisfying, easily kept by the resident doctors as a treatment package for bronchiolitis, and easily carried out by the nursing staff.

The treatment expenses are found to be much less when antibiotics, $\beta 2$ agonist, and steroids are omitted. 


\section{Discussion}

Of note, there is no similar study on contributory factors to acute bronchiolitis, the prevalence of acute bronchiolitis is increasing in port Sudan city than what we used to see previously, primarily due to the changing lifestyle and living in flats with decreased ventilation, and many other contributory factors, the most prominent being the avoidance of bathing infants during the winter months (due to the widely spread believe in the Arabic world that bathing increases respiratory diseases, in addition to the unawareness of the mothers and carers of the importance of hand washing). Residing in flats which lack good ventilation and sun is an important factor that encourages viruses to live and persist in the place. The previous Sudanese housing models of large houses with compounds and good ventilation allowed sun exposure and were therefore unsuitable for viruses to live or persist. This style has now changed to apartments and flats. Other factors like poor self-hygiene due to illiteracy, the presence of a smoking person in the household, and over-extending families living in the same place or the crowding of the Sudanese social activity play major roles in increasing acute bronchiolitis prevalence in port Sudan city. Though the incidence is large in the underdeveloped countries despite the incomplete data according to WHO [13], fortunately, the prevalence of severe cases in Sudan is much less compared to other countries like Egypt and KSA (unpublished observational study). The new protocol showed simplicity and acceptance and effectiveness. No encountered complications nor death, and expenses including length of hospital stay were significantly less, that is because bronchiolitis is a viral disease and the use of antibiotics and steroids will decrease the immunity (killing the normal commensals and suppressing the immune system) subjecting the recipient to more spread of the virus and hence more prolonged and severe disease. The use of $\beta_{2}$ agonist is controversial, as there is no element of bronchospasm in the pathogenesis of bronchiolitis, but it may help thinning the mucus to ease its excretion. Using the stated clinical classifying criteria allows diagnosis acute bronchiolitis on clinical basis only, suits poor-resourced places, and is in agreement of discouraging unnecessary investigation and unneeded treatment [14].

\section{Conclusion}

Acute bronchiolitis prevalence is increasing in our city, the Reg- 1 and 2 regimens should be adopted as a hospital policy in treating bronchiolitis as it proved its efficacy, safety, and cheapness.

\section{Acknowledgements}

The author is greatly indebted to all doctors and nursing staff in the pediatric ward of our hospital who helped with the current study, as well as to all parents and carers who gave their trust and acknowledged the way of treatment. 


\section{Ethical considerations}

This study was approved by the SPCH ethical committee on September 8, 2013.

\section{References}

[1] Medline Plus. (2020). Medline Plus Medical Encyclopedia: Bronchiolitis. Retrieved from http://www.nlm. nih.gov/medlineplus/ency/000975.htm

[2] NSW. (2018). Management of Acute Bronchiolitis. Retrieved from http://www.eih.health.nsw.gov.au/ _data/assets/pdf_file/0010/458884/Clinical-practice-guideline-acute-management-of-bronchiolitis. pdf

[3] Piedra, P. A. and Stark, A. R. (2013). Bronchiolitis (and RSV) in infants and children (Beyond the Basics) in UpToDate.

[4] The Royal Children's Hospital Melbourne. Clinical Guidelines: Bronchiolitis. Retrieved from http://www. rch.org.au?rchcpg/hospital_clinical_guidelines

[5] Fujiogi, M., Goto, T., Yasunaga, H., et al. (2013). Trends in bronchiolitis hospitalizations in the United States, 2000-2009. Pediatrics, vol. 132, no. 1, pp. 28-36.

[6] Hall, C. B., Weinberg, G. A., Blumkin, A. K., et al. (2013). Respiratory syncytial virus-associated hospitalizations among children less than 24 months of age. Pediatrics, vol. 132, no. 2, pp. e341-e348.

[7] Basco, W. T., Jr. (August 23, 2013). Infants with bronchiolitis: what treatment is best? Medscape Pediatrics.

[8] Shay, D. K., Holman, R. C., Newman, R. D., et al. (1999). Bronchiolitis-associated hospitalizations among US children, 1980-1996. JAMA, vol. 282, pp. 1440-1446.

[9] Nagakumar, P. and Doull, I. (2012). Current therapy for bronchiolitis. Archives of Disease in Childhood, vol. 97, no. 9, pp. 827-830.

[10] Midulla, F. (2010). Respiratory syncytial virus, human bocavirus and rhinovirus bronchiolitis in infants. Archives of Disease in Childhood, vol. 95, pp. 35-41.

[11] Brandenburg, A. H. (1997). Local variability in respiratory syncytial virus disease severity. Archives of Disease in Childhood, vol. 77, no. 5, pp. 410-414.

[12] Kneyber, M. C., Brandenburg, A. H., Rothbarth, P. H., et al. (1996). Relationship between clinical severity of respiratory syncytial virus infection and subtype. Archives of Disease in Childhood, vol. 75, no. 2, pp. 137-140.

[13] Rudan, I., Tomaskovic, L., Boschi-Pinto, C., et al. (2004). Global estimate of the incidence of clinical pneumonia among children under five years of age. Bulletin of the World Health Organization, vol. 82, no. 12 , pp. 895-903.

[14] Schroeder, A. R. and Mansbach, J. M. (2014). Recent evidence of management of bronchiolitis. Current Opinion in Pediatrics, vol. 26, no. 3, pp. 328-333. 